\title{
Evaluación de los Componentes del Somatotipo e Índice de Masa Corporal en Escolares del Sector Precordillerano de la IX Región, Chile
}

\author{
Evaluation of the Somatotipe Components and Body Mass Index in Scholarships of \\ Foothills at the IX Región, Chile
}

"Héctor Silva M.; "Erika Collipal L.; ** Cristian Martínez \& ***José Bruneau C.

SILVA, M. H.; COLLIPAL, L. E.; MARTINEZ, C. \& BRUNEAU, C. J. Evaluación de los componentes del somatotipo e índice de masa corporal de niños del sector precordillerano de la IXRegión, Chile. Int. J. Morphol., 23(2):195-199, 2005.

RESUMEN: La obesidad en el mundo se ha convertido en un problema de salud pública, no escapando a esta situación los adolescentes de Chile. Los estudios de la composición corporal, estado nutricional e índice de masa corporal (IMC) son los parámetros recomendados por la OMS, para determinar el estado nutricional. Realizamos este estudio para conocer la realidad del sector cordillerano de la Novena Región de Chile.

Evaluamos 662 adolescentes, 358 hombres (54,1\%) y 304 mujeres (45,9\%), entre 11 y 15 años de edad, alumnos de colegios urbanos municipalizados del sector Precordillerano de la Novena Región Chile. Para obtener el somatotipo utilizamos el método antropométrico de Heath \& Carter, efectuándose la clasificación del IMC de acuerdo a las normas de la OMS.

Los hombres, eran más mesomórficos que las mujeres, siendo esta diferencia estadísticamente significativa. Los componentes endomórfico y ectomórfico no presentaron diferencias estadísticamente significativas de acuerdo al sexo. El 50,8\% de los hombres y el $34,1 \%$ de las mujeres presentaban un IMC normal. Observamos un predominio del componente mesoendomórfico. Los individuos presentaron obesidad en el $2,7 \%$ y sobrepeso en el $10,7 \%$ de los casos.

Estos resultados revelan una prevalencia de obesidad baja si son comparados con otros estudios efectuados en Chile, siendo posiblemente la alta ruralidad, la pobreza, la alimentación y los diferentes estilos de vida los que han influido en estos resultados.

PALABRAS CLAVE: Somatotipo; Antropometría; Índice de masa corporal.

\section{INTRODUCCIÓN}

Aplicando técnicas antropométricas se ha demostrado que existen distintos patrones de distribución del somatotipo en las diferentes etapas de la vida. Has ta hace algunos años, las evaluaciones de los estados de crecimiento y nutrición se investigaban, fundamentalmente, en relación con curvas de crecimiento. Estos estándares solo consideraban peso y talla, a partir de los cuales se infería el grado de retraso, normalidad y adelanto del crecimiento. Además, fueron usados para saber si los niños eran obesos o estaban desnutridos en relación a su altura.
El análisis de los pliegues cutáneos permite estimar el contenido de masa grasa y masa libre de grasa en los diferentes individuos y, consecuentemente, inferir los requerimientos nutricionales. Toro, \& Almagià, (1989, 1994); Depress (1997); Swan \& McConnell (1999).

El somatotipo refleja los constituyentes del cuerpo y permite determinar su endomorfía, (cantidad de tejido adiposo), mesomorfía (masa muscular) y ectomorfía (linealidad). Por otra parte, los estudios han indicado que en

\footnotetext{
* Dpto. de Ciencias Básicas, Facultad de Medicina, Universidad de La Frontera, Chile.

** Dpto. de Educación Física, Facultad de Educación y Humanidades, Universidad de La Frontera, Chile.

Centro de Alto rendimiento, IX Región de Chile
} 
la adolescencia las mujeres son más endomórficas que los hombres. Almagià et al. (1996a) en un estudio de corte transversal encontró, en la mayoría de los casos, un componente mesomórfico balanceado.

En el último decenio en Chile la talla de los adolescentes ha tendido a un cambio positivo, Burrow et al. (1998). Éste está relacionado con factores genéticos y ambientales, influyendo también el índice de masa corporal (IMC) de la madre al momento de la preconcepción. Así, mujeres con IMC y talla bajos, paren hijos con bajo peso y con retardo de crecimiento intrauterino. Bolzan \& Guimarey (2001); Lagos et al. (1999); Mardones et al. (1999); De Paoli et al. (2001). Según Erazo, et al. (1998), estos niños podrían tener en el futuro un menor rendimiento escolar.

Según Almagià et al. (1997), el cambio en la dieta que se ha producido en Chile y el aumento de la obesidad, se ha convertido en un problema dramático en la población. La prevalencia de obesidad en Chile era, entre los años 1987 y 1996, de 13,4\% para hombres y de un $15 \%$ para mujeres y el sobrepeso de $19 \%$ en hombres y $17,2 \%$ en mujeres según Atalah et al. (1999); Valenzuela (2002).

Los factores que han producido el cambio del IMC en los últimos decenios, son producto del sedentarismo y del creciente consumo de grasas y azúcares. Estos elementos contribuyen a un aumento de la obesidad, hiperlipidemias, caries dentarias, diabetes, cáncer, osteoporosis y otras enfermedades crónicas, incluso se asocian a telarquia aislada sin vello púbico, que ha sido descrito en otras poblaciones. Burrows et al. (1988); Codner (2004).

Según Bustos (2003) en un estudio realizado en adultos jóvenes, en la ciudad de Limache, Chile, relacionado con factores de riesgo de enfermedades cardiovasculares, llamó la atención las elevadas cifras de obesidad y sobrepeso que comprometían a casi el 50\% de esta población, incluso $30,8 \%$ de ellos, presentaba un mayor acúmulo de grasa abdominal.

Los estudios de obesidad y del IMC han sido escasos en la IX Región de Chile. Según el Ministerio de Salud de Chile, existe un 25\% de sobrepeso y obesidad en menores de 6 años, controlados en el Sistema Nacional de Servicios de Salud, un 35\% en preescolares que asisten a Jardines Infantiles del Gobierno, un 38\% en escolares de 1er año Básico, un 33\% en escolares púberes y cerca de un $50 \%$ en adultos, dependiendo de la edad y sexo. Por otro lado, 40 a $76 \%$ de los escolares obesos serán adultos obesos. Dada la alta prevalencia de sobrepeso y obesidad en escolares y adolescentes en Chile, nos parece necesario implementar la evaluación periódica de este grupo etario, el que tradicionalmente no se controla regularmente.

Con relación a la situación nutricional por exceso (indicador peso para la talla $>+1$ y $+2 \mathrm{DE}$ ) se observa que el porcentaje de menores con sobrepeso y obesidad en Chile, ha ido en aumento, de $22,4 \%$ y de $9,4 \%$ en 1996 a $23 \%$ y $10,6 \%$ en el 2000. En el ámbito nacional se observa una mayor magnitud de éstas en las Regiones III, X y XII.

Jadue et al. (1999) al analizar de obesidad según nivel socioeconómico, encontró una prevalencia de 28,9\% en mujeres y $18,6 \%$ en hombres de nivel socioeconómico bajo. Más del $90 \%$ de las mujeres no hacían ejercicio en el tiempo libre y en los hombres este problema aumentaba con la edad.

La Encuesta Nacional del Deporte de 1996, en una muestra de 4300 personas entre 10 y 70 años, reveló que el $83 \%$ de los individuos eran sedentarios, la que afectaba con mayor intensidad a mujeres de nivel socioeconómico bajo.

Por otra parte, en la población infantil, se ha demostrado que los juegos electrónicos y la educación pasiva, contribuyen de manera importante a disminuir el gasto energético. Los niños obesos y normales, durante la semana gastan más de tres horas diarias frente al televisor, siendo cuatro horas en los días festivos.

Con la finalidad de poder detectar los niveles de obesidad, que afectan a la población adolescente del sector Precordillerano de la IX Región de Chile, realizamos un estudio del somatotipo e índice de masa corporal.

\section{MATERIAL Y MÉTODO}

Evaluamos el somatotipo e índice de masa corporal en 662 adolescentes, 358 hombres $(54,1 \%)$ y 304 mujeres (45,9\%), entre 11 y 15 años de edad, alumnos de colegios urbanos municipalizados del sector Precordillerano de la IX Región, Chile. Los sujetos fueron evaluados en sus colegios y los datos registrados en una ficha.

Los instrumentos de medición utilizados fueron: Banco de antropometría, estadíometro de pared, adipómetro de Harpender, antropómetro de Lafayette y cinta métrica Sany.

Para la obtención del somatotipo utilizamos el método de Heath \& Carter (1967) y calculamos el índice de masa corporal según OMS. Empleamos el programa SPSS 10.0 para el análisis de los datos. 


\section{RESULTADOS}

Los valores de los componentes del somatotipo e índice de masa corporal se encuentran en las tablas I a IV.

Tabla I. Clasificación del somatotipo en adolescentes de ambos sexos, según edad del sector Precordillerano de la IX Región, Chile.

\begin{tabular}{ccccccc}
\hline Edad (años) & \multicolumn{2}{c}{ Endomorfía } & \multicolumn{2}{c}{ Mesomorfía } & \multicolumn{2}{c}{ Ectomorf ía } \\
& Hombres & Mujeres & Hombres & Mujeres & Hombres & Mujeres \\
11 & 4.6 & 4.3 & 5.3 & 4.2 & 2.2 & 2.9 \\
12 & 4.4 & 5.4 & 5.0 & 6.0 & 2.4 & 2.2 \\
13 & 4.5 & 5.2 & 5.7 & 4.1 & 2.3 & 2.4 \\
14 & 4.1 & 5.8 & 5.0 & 4.1 & 2.7 & 2.1 \\
15 & 3.9 & 5.6 & 4.8 & 5.2 & 2.6 & 1.8 \\
\hline
\end{tabular}

Tabla II. Clasificación del somatotipo según promedio por edad en adolescentes, de ambos sexos, del sector Precordillerano de la IX Región, Chile.

\begin{tabular}{cccc}
\hline Edad (años) & Endomorfía & Mesomorfía & Ectomorfía \\
11 & 4.5 & 4.9 & 2.4 \\
12 & 4.9 & 5.5 & 2.3 \\
13 & 4.9 & 4.8 & 2.3 \\
14 & 4.6 & 4.7 & 2.5 \\
15 & 4.7 & 5.0 & 2.3 \\
\hline
\end{tabular}

Tabla III. Distribución del índice de masa corporal en adolescentes según edad y sexo del sector Precordillerano de la IX Región, Chile.

\begin{tabular}{lcccc}
\hline Edad & $\begin{array}{c}\text { X IMC } \\
\text { Hombres }\end{array}$ & DS & $\begin{array}{c}\text { X IMC } \\
\text { Mujeres }\end{array}$ & DS \\
11 & 17,04 & 1 & 17,2 & 1,5 \\
12 & 18,9 & 1,4 & 19,5 & 0,9 \\
13 & 20,1 & 1,7 & 19,7 & 1,0 \\
14 & 19,5 & 1,9 & 21,5 & 1,0 \\
15 & 20,5 & 1,2 & 22,3 & 0,9 \\
\hline
\end{tabular}

Tabla IV. Distribución del índice de masa corporal en adolescentes de ambos sexos, del sector precordillerano de la IX Región, Chile

\begin{tabular}{lcccrrrrr}
\hline Sexo & \multicolumn{3}{c}{ Bajo peso } & \multicolumn{2}{c}{ Normal } & \multicolumn{2}{c}{ Sobre peso } & \multicolumn{2}{c}{ Obeso } \\
& $\mathrm{N}$ & $\%$ & $\mathrm{~N}$ & $\%$ & $\mathrm{~N}$ & $\%$ & $\mathrm{~N}$ & $\%$ \\
& & & & & & & & \\
Hombres & 7 & 1,1 & 336 & 50,8 & 7 & 1,1 & 8 & 1,2 \\
Mujer & 4 & 0,6 & 226 & 34,1 & 64 & 9,7 & 10 & 1,5 \\
Total & 11 & 1,7 & 562 & 84,9 & 71 & 10,7 & 18 & 2,7 \\
\hline
\end{tabular}

\section{DISCUSIÓN}

El somatotipo permite evaluar biométricamente a los individuos en sus componentes endomórficos, mesomórficos y ectomórficos que representan los tejidos primarios.

Es este estudio la mesomorfía fue mayor en los varones y la endomorfía en las mujeres, siendo estas diferencias estadísticamente significativas, concordando con otros estudios realizados por Toro et al. (1989, 1994); Almagià et al. (1996b) y Silva et al. (2003).

Se puede observar que a los 12 años las mujeres presentan una mesomorfía mayor que los hombres, posiblemente determinado por el desarrollo fisiológico más temprano en las mujeres.

Con relación al total de la muestra, los valores de mesomorfía en promedio son más altos que los valores de endomorfía, siendo esta diferencia estadísticamente significativa.

El IMC es un indicador que mide el estado nutricional del individuo. Como la obesidad ha ido en aumento en nuestro país, según el Ministerio de Salud de Chile (MINSAL, 2003) y diversas investigaciones realizadas por el Instituto de Nutrición y Tecnología de los Alimentos de Chile (INTA), conocer la realidad de los adolescentes en esta Región de Chile es importante para las futuras políticas de Salud del país.

Tanto en los hombres como en las mujeres el IMC se encuentra dentro del rango normal, pero muy próximas al sobrepeso. Sin embargo, los índices de sobrepeso y obesidad en escolares del sector precordillerano no superaron el 13, $4 \%$.

Según estudios y encuestas realizadas por el MINSAL, existe un 33\% de sobrepeso y obesidad en escolares púberes de la IX Región.

Es necesario destacar que los resultados obtenidos en este estudio, en relación a las variables bajo peso y sobrepeso, son diferentes a los resultados publicados por el MINSAL (2001).

Los valores obtenidos de sobrepeso de 10,7\% y obesidad de $2,7 \%$, en adolescentes menores de 12 
años, son inferiores a los reportados por el MINSAL (sobrepeso, 16,5\% obesidad 13,5 de la IX Región de Chile), debido probablemente a que estos últimos realizaron sus estudios en las grandes ciudades del país, incluyendo Temuco, donde los hábitos alimenticios de los adolescentes son muy diferentes a los de nuestro estudio.

Por lo anterior y teniendo en consideración que la IX Región presenta los mayores rangos de pobreza, los bajos índices de obesidad obtenidos en este estudio, se explicarían debido a diversos factores tales como: ruralidad, tipo de alimentación, actividades recreativas de los estudiantes, menor acceso a entretención de tipo tecnológica (juegos nintendo, computadores, videos) y, finalmente, menor acceso a una alimentación con alto contenido de materia grasa, lo que en definitiva, lo diferencia de los grandes centros urbanos, donde estas variables son más asequibles para todos los niveles socioeconómicos.

SILVA, M. H.; COLLIPAL, L. E.; MARTINEZ, C. \& BRUNEAU, CH. J. Evaluation of the somatotipe components and body mass index in scholarships of foothills at the IX Región, Chile. Int. J. Morphol., 23(2):195-199, 2005.

SUMMARY: World obesity has become a public health problem, Chilean adolescents do not escape this situation. Studies on body composition, nutritional state, and body mass index (BMI), are parameters recommended by the WHO for determining human beings' nutritional state. This study has been carried out to know the real of situation of adolescents living in the foothills of the Andes, in the IX Región in Chile.

Six hundreds and sixty two subjects were evaluated, 358 men $(54,1 \%)$ and 304 women $(45,9 \%)$, between 11 and 15 years old, all students at urban municipal public schools, in the foothills of the Andes, in the IX Region of the Chilean Araucanía. In order to obtain the somatotype the anthropometric Health \& Carter method was used, classifying BMI according to the WHO norms.

The sample was mesoendomorphofic and indicated that the females were more endomorphic that males, being this difference statistically significant. The endomorphic and ectomorphic components presented no statically significant differences according to sex. The MBI showed values of $47 \%$ higher than the norm in males and of $33 \%$ over the norm in females. The sample presented a predominance of the mesomorphic component.

The obesity rate was $2,7 \%$ and overweight was $10,7 \%$. These results showed low prevalence of obesity contrasted with the national rate, the reasons could be rurality, poverty, food, and different life style in relation to city life.

KEY WORDS: Somatotype; Anthropometry; Body mass index.

\section{REFERENCIAS BIBLIOGRÁFICAS}

Almagià, A.; Toro, T.; Gurovich A.; Cabrera, E.; Marinao, A. \& Binvignat, O. Determinación y análisis de la morfoestructura de un equipo de fútbol profesional. Rev. Chil. Anat., 14(1):87-95, 1996a.

Almagià, A.; Toro, T.; Binvignat, O.; Cabrera, E. \& Marinao, A. Aproximación al perfil morfoestructural y dimorfismo sexual de jóvenes de ambos sexos de 6 a 23 años de edad caracterizados por el somatotipo. Rev. Chil. Anat., 14(2):189-97, 1996b.

Almagià, F. A. A.; Gurovich, M. A.; Ivanovic, M. D.; Toro, D.T. \& Binvignat, G. O. Estudio y análisis morfológico etario del dismorfismo sexual a través de la composición corporal. Rev. Chil. Anat., 15(2):141-9,1997.

Atalah, E.; Arteaga, C. Rebolledo, A.; Delfín S. \& Ramos, R. Prevalencia de obesidad en escolares de la Región de Aysén. Rev. Chil. Pediatr., 70(3):208-14,1999.
Bolzan, A. \& Guimarey, L. Relación entre el índice de masa corporal durante la gestación en embarazadas adolescentes y adultas, indicadores antropométricos de crecimiento fetal y retardo de crecimiento intrauterino. Arch. Lat. Nutric., 51(4): 99-103, 2001.

Burrows, R.; Leiva, L.; Mauricci, A.; Zvaighaft, A. \& Muzzo, S. Características de la pubertad de niñas escolares de la Región Metropolitana. Rev. Chil. Pediatr., 59(2):215, 1998.

Burrows, R.; Ramírez, I.; Cordero, J. \& Muzzo, S. Cambio secular del retraso de talla en escolares chilenos de tres regiones del país. Rev. Chil. Pediatr., 70(5):390-7, 1998.

Bustos, M. P.; Amigo, C. H.; Arteaga, L. A.; Acosta, B. A. M. \& Rona, R. J. Factores de riesgo de enfermedad cardiovascular en adultos jóvenes. Rev. Méd. Chile., 131(9):973-980, 2003. 
Crovetto, M. Cambios en la estructura alimentaría y consumo aparente de nutrientes de los hogares del Gran Santiago 1988-1997. Rev. Chil. Nutr,. 29(1):24-32, 2002.

Depress, P. Visceral obesity, insulin resistance and dyslipidemia: contribution of endurance exercise training to the treatment of the plurimetabolic syndrome. Exercise and Sport Scienc., 25:271-300, 1997.

De Paoli, R.; Aguaje, A. \& Henríquez, G. Cambios en las variables antropométricas durante la gestación en mujeres eutróficas. Arch. Lat. Nutric., 51(4):351-9, 2001.

Erazo, M.; Amigo, H.; De Andraca, I. \& Bustos, P. Déficit de crecimiento y rendimiento escolar. Rev. Chil. Pediatr., 69(3):94-8,1998.

Jadue, C.; Vega, M. J.; Escobar, S. M.; Delgado, B. I.; Garrido, G. C.; Lastra, M. P.; Espejo, E. F. \& Peruya, V. A. Factores de riesgo para las enfermedades no trasmisibles; Metodología y resultados globales de la encuesta de base del programa CARMEN. Rev. Med. Chile, 127:1004$13,1999$.

Lagos, R.; Espinoza, R.; Orellana, J. \& Echeverría, P. Diferencia en peso de nacimiento promedio según tres variables biológicas en recién nacidos normales. Rev. Med. Chile. 127(12):1425-30, 1999.

Mardones, F.; Bastías, G.; Farías, M.; Dinamarca, R.; Olavaria, F.; Rada, G.; Rojas, A. \& Rojas, P. Composición corporal de neonatos con alteraciones en el crecimiento fetal. Rev. Chil. Pediatr., 70(4):300-5, 1999.

MINSAL, Situación epidemiológica nutricional de Chile. h t t p : / / w w w. mi n s a l. c l/ i c i / n u t ricion / Mapa\%20Nut\%20Chile\%20FAO.pdf, 2001.

MINSAL. Norma técnica de evaluación nutricional del niño de 6 a 18 años. http://www.minsal.cl/ici/nutricion\%5 CNormaEvNut6a18anos.pdf, 2003.

Swan, P. \& McConnell, K. Anthropomety and bioelectrical impedance inconsistently predicts fatness in women with regional adiposity. Med. Scienc. Sport. Exerc., 31(7):1068-75, 1999.

Silva, H.; Bruneau, J. C.; Reyno, H. P. et al. Somatotipo e índice de masa corporal en una muestra de adolescentes de ambos sexos de la ciudad de Temuco, Chile. Int. J. Morphol., 21(4):309-13. 2003

Toro, T. \& Almagià, A. Aplicación de estándares antropométricos para la evaluación del crecimiento y estado nutricional en niñas y niños de 11-15 años de Valparaíso. An. Anat. Nor., 7:99-107, 1989.

Toro, T. \& Almagià, A. Mediciones biométricas, incluyendo grosor de pliegues cutáneos como indicadores de la composición corporal y estado nutricional. Estudio longitudinal en escolares de Valparaíso. Rev. Chil. Anat., 12(2):197-204, 1994.

Valenzuela, A. Obesidad. 2. ed. Mediterráneo, Santiago, 2002.

Dirección para correspondencia:

Prof. Héetor Silva Mella

Facultad de Medicina

Universidad de La Frontera

Casilla $54-\mathcal{D}$

Temuco - CHILE

Recibido : 12-01-2005

Aceptado: 22-05-2005 